Pacific Journal of Mathematics

PROJECTIVE SPACE AS A BRANCHED COVERING WITH 


\title{
PROJECTIVE SPACE AS A BRANCHED COVERING WITH ORIENTABLE BRANCH SET
}

\author{
ROBERT D. LitTLE
}

\begin{abstract}
We show that if $R P^{n}$ is a smooth $k$-fold branched covering of $S^{n}$ with orientable branch set and either $k \leqq n$ or the singular set of the covering is connected, then $n=$ 1,3 , or 7 .
\end{abstract}

1. Introduction. Let $\tilde{M}$ and $M$ be smooth, closed $n$-manifolds. A $k$-fold branched covering is a smooth map $f: \widetilde{M} \rightarrow M$ together with smoothly imbedded, codimension 2 submanifolds $K \subset M$ and $\widetilde{K}=f^{-1}(K) \subset \widetilde{M}$, such that $f$ restricted to $\tilde{M}-\widetilde{K}$ is a $k$-fold covering, $f$ restricted to $\widetilde{K}$ is a finite covering onto each component of $K$, and the degree of $f$ is $k$. Brand [2] suggests the problem of determining the values of $n$ for which $\boldsymbol{R} P^{n}$, real projective $n$-space, is a branched covering of $S^{n}$ and he shows that if $R P^{n}$ is a branched covering of any manifold with trivial Stiefel-Whitney class, such as $S^{n}$, then $n=2^{t} \pm 1$. We show that the possible values of $n$ can be further limited if the branch set of the covering, $K$, is orientable and either $k \leqq n$ or the covering is simple, that is, the singular set, the subset of $\widetilde{K}$ where $f$ fails to be a local diffeomorphism, is connected.

THEOREM 1.1. If $\boldsymbol{R} P^{n}$ is a $k$-fold branched covering of $a \pi$ manifold with orientable branch set and either $k \leqq n$ or the covering is simple, then $n=1,3$, or 7 .

A $\pi$-manifold is a smooth, closed $n$-manifold with stably trivial tangent boundle, such as $S^{n}$. Our definition of simple branched covering is due to Hilden, [5]. Brand and Brumfiel have extensively studied simple branched coverings having the special property that the connected singular set is all of $\widetilde{K},[3]$.

The identity map provides a branched covering of $S^{1}$ by $R P^{1}$ and it is well known that $R P^{3}$ is a 2 -fold branched covering of $S^{3}$. Hilden and Montesinos have shown, independently, that every closed orientable 3-manifold is a simple, 3-fold branched covering of $S^{3}$, [5], [7].

2. Normalized branched coverings. In [2], Brand defines a normalized branched covering. He uses this concept to show that there is a certain $K$-theoretic necessary condition for the existence of a branched covering. 
If $\eta$ is a 2-plane bundle over a complex $X$, let $\mu_{k}(\eta)$ be the 2-plane bundle obtained from $\eta$ by the homomorphism $\mu_{k}: O(2) \rightarrow$ $O(2)$ given by $\mu_{k}(z)=z^{k}$ for $z \in S O(2)$ and $\mu_{k}(\tau)=\tau$ for $\tau=\left(\begin{array}{rr}1 & 0 \\ 0 & -1\end{array}\right)$. If $k$ is odd, $\mu_{k}$ and the Adams operation $\psi^{k}$ agree on 2-plane bundles, ([1], p. 193). There is a map, $\mu_{k}: \eta \rightarrow \mu_{k}(\eta)$, given in local coordinates by $\mu_{k}(x, z)=\left(x, z^{k}\right)$, where $x \in X$ and $z \in \boldsymbol{R}^{2}=\boldsymbol{C}$. The definition which follows is due to Brand, [2].

DEFinition 2.1. A branched covering $f: \widetilde{M} \rightarrow M$ is called a normalized branched covering if there are tubular neighborhoods $\widetilde{N}$ and $N$ of $\widetilde{K}$ and $K$, respectively, with the following properties:

2.2. $d f_{x}$ is non-singular for each $x \in \widetilde{M}-\widetilde{K}$,

2.3. $d f \mid \widetilde{K}: \tau \widetilde{K} \rightarrow \tau K$ is an isomorphism on each fibre and

2.4. if $\widetilde{\xi}=$ normal bundle of $\widetilde{K} \subset \widetilde{M}$ and $\xi=$ normal bundle of $K \subset M$, then for each component $\widetilde{K}_{i}$ of $\widetilde{K}$, there is an integer $k_{i}$ such that $\mu_{k_{i}}(\tilde{\xi})\left|\widetilde{K}_{i} \cong f * \tilde{\xi}\right| \widetilde{K}_{i}$ and the map $f \mid \widetilde{N}$, mapping $\widetilde{N}$ onto $N$, is given by $D \tilde{\xi}\left|\widetilde{K}_{i} \stackrel{\mu_{k_{i}}}{\longrightarrow} D \mu_{k_{i}}(\tilde{\xi})\right| \widetilde{K}_{i} \cong D f * \xi \mid \widetilde{K}_{i} \stackrel{f^{*}}{\longrightarrow} D \xi$.

It is possible to visualize a $k$-fold, normalized branched covering in the following way. For each component $K_{j}$ of the branch set $K$, there is associated a partition of $k, \sum_{i=1}^{n_{j}} l_{i} k_{i}=k$, where the number of components of $f^{-1}\left(K_{j}\right)$ is $\sum_{i=1}^{n_{j}} l_{i}$, and on $l_{i}$ of these components, the map $f$ has the form $f(x, z)=\left(f(x), z^{k_{i}}\right)$ in the local coördinates of the disc bundle of the normal bundle of $\widetilde{K}$, [2]. The integers $k_{i}$ are called the exponents of the branched covering. Each exponent is less than or equal to $k$, and so a normalized branched covering has only a finite number of distinct exponents. Notice that a simple branched covering has only one exponent greater than or equal to 2. In the case of the Hilden-Montesinos simple coverings of $S^{3}, K$ is a knot and $f^{-1}(K)$ consists of two components, one of which, the singular set, has exponent 2, [5].

For each integer $k \geqq 2$, Brand defines a $K$-theoretic characteristic class $\eta_{k}$ in $K O(M O(2))$ with the property that $\eta_{k} \mid B O(2)=\gamma-\mu_{k}(\gamma)$, where $\gamma$ is the universal 2-plane bundle. Let $\widetilde{K}_{k_{i}}=\{x \in \widetilde{K}: f$ maps the fibre over $x$ of the tubular neighborhood of $\widetilde{K}$ in $\widetilde{M}$ to the fibre over $f(x)$ of the tubular neighborhood of $K$ in $M$ by the map $z \rightarrow z^{k_{i}}$, where $k_{i}$ is one of the exponents of $f, 1 \leqq i \leqq m$. For each $i$, $1 \leqq i \leqq m$, let $g_{i}$ be the composite map

$$
M \stackrel{c_{i}}{\longrightarrow} T\left(\tilde{\xi} \mid \widetilde{K}_{k_{i}}\right) \stackrel{h_{i}}{\longrightarrow} M O(2),
$$

where $c_{i}$ is the collapsing map onto the Thom space and $h_{i}$ is induced by the classifying map of the bundle $\tilde{\xi} \mid \widetilde{K}_{k_{i}}$. A slight modification in the proof of Brand, taking into account the fact that the number 
of distinct exponents is finite, yields formula (2.7) in the next theorem.

THEOREM 2.6. (Brand, [2]) If $f: \widetilde{M} \rightarrow M$ is a branched covering, then, given a differential structure on $M$, there is a unique differential structure on $\widetilde{M}$ making $f$ a normalized branched covering. The tangent bundle of $\widetilde{M}$ determined by this unique differential structure is related to the exponents of $f$ by an equation in the group $\widetilde{K O}(M)$,

$$
\tau \tilde{M}-f^{*} \tau M=\sum_{i=1}^{m} g_{i}^{*} \eta_{k_{i}}
$$

We will conclude this section by proving a theorem which shows that (2.7) does not depend on the change of differential structure in the special case $\widetilde{M}=\boldsymbol{R} P^{n}$ and that this formula is especially simple in the case where the branch set is orientable. Before we can state and prove this theorem, we need some preliminaries, beginning with a lemma about smoothings of $\boldsymbol{R} \boldsymbol{P}^{n}$. By a smoothing of $\boldsymbol{R} \boldsymbol{P}^{n}$, we mean a smooth $n$-manifold, $M$, together with simple homotopy inverses $\psi: \boldsymbol{R} P^{n} \rightarrow M$ and $\varphi: M \rightarrow \boldsymbol{R} P^{n}$. We may assume that $\varphi$ and $\psi$ are homotopy equivalences only, since $W h\left(\boldsymbol{Z}_{2}\right)=0$, ([4], pp. 45 and 72).

LEMMA 2.8. If $\psi: R P^{n} \rightarrow M$ and $\psi^{\prime}: R P^{n} \rightarrow M^{\prime}$ are two smoothings of $\boldsymbol{R} P^{n}$, then in $K O\left(\boldsymbol{R} P^{n}\right), \psi^{*}(\tau M)=\psi^{\prime *}\left(\tau M^{\prime}\right)$.

Proof. We may assume that the twisted degree of $\phi$ is +1 , since we may compose with a self homotopy equivalence of $\boldsymbol{R} P^{n}$ of degree -1 in the case $n$ odd where twisted degree is the ordinary degree ([8], p. 466), and since $\operatorname{deg} \varphi=+1$ necessarily in the case $n$ even, ([8], p. 468). By setting $\nu=\psi^{*} \nu_{M}$, where $\nu_{M}$ is the stable normal bundle of $M$, we see that a smoothing determines an element of the bordism group $\Omega_{n}\left(\boldsymbol{R} P^{n}, \nu\right)$, ([9], p. 32), of degree +1 . The smoothing $M^{\prime}$ determines an element of degree +1 in $\Omega_{n}\left(\boldsymbol{R} P^{n}, \nu^{\prime}\right)$, where $\nu^{\prime}=\psi^{\prime} \nu_{M^{\prime}}$. Let $\nu_{R P^{n}}$ be the Spivak normal fibration of $\boldsymbol{R} \boldsymbol{P}^{n}$, ([9], p. 105). According to Spivak's theorem, $\Omega_{n}\left(\boldsymbol{R} P^{n}, \nu\right)$ contains an element of degree +1 if, and only if, $\nu$ is stably fibre homotopy equivalent to $\nu_{R P^{n}}$. Therefore, $\nu$ and $\nu^{\prime}$ are stably fibre homotopy equivalent, that is, they have the same image under the map $\widetilde{K O}\left(\boldsymbol{R} P^{n}\right) \rightarrow \widetilde{K}_{\text {Top }}\left(\boldsymbol{R} P^{n}\right)=$ the group of stable fibre homotopy equivalences classes. But image $\left\{\widetilde{K O}\left(\boldsymbol{R} P^{n}\right) \rightarrow \widetilde{K}_{\text {Top }}\left(\boldsymbol{R} P^{n}\right)\right\}=J\left(\boldsymbol{R} P^{n}\right)$, ([6], p. 211), and $\widetilde{K O}\left(\boldsymbol{R} P^{n}\right) \cong J\left(\boldsymbol{R} P^{n}\right)$, ([6], p. 225). Therefore, $\nu$ and $\nu^{\prime}$ are stably isomorphic. It follows easily that $\psi^{*}(\tau M)=\psi^{\prime *}\left(\tau M^{\prime}\right)$. 
If $\boldsymbol{R} \boldsymbol{P}^{n}$ is a branched covering of a $\pi$-manifold, then it follows from the full generality of the theorem of Brand that $n=2^{t} \pm 1$. If the branch set $K$ is orientable, so is $\widetilde{K}$, since $f \mid \widetilde{K}$ is a finite covering, and therefore $\tilde{\xi}$ is orientable because $R P^{n}$ is orientable when $n$ is odd. This means that the maps $h_{i}: T\left(\widetilde{\xi} \mid \widetilde{K}_{k_{i}}\right) \rightarrow M O(2)$ lift to $M S O(2)$, which has the same homotopy type as $B S O(2)$ and $B U(1)$. These liftings are the only consequences of the orientability of $K$ needed in the proof of Theorem 1.1, and so we remark that Theorem 1.1 remains true if the hypothesis that $K$ is orientable is replaced by the weaker hypothesis that $\widetilde{K}$ is orientable.

It follows from the construction of Brand that there are orientable characteristic classes $\eta_{k}$ in $K O(M S O(2))$, such that $\eta_{k} \mid B S O(2)=$ $\gamma-\mu_{k}(\gamma)$, where $\gamma$ is the universal orientable 2-plane bundle, ([2], p. 2). Using the same symbols in the orientable and non-orientable cases will not cause any confusion in what follows.

The group $\widetilde{K O}\left(\boldsymbol{R} P^{n}\right)$ is a finite cyclic group with generator $\lambda-1$, where $\lambda$ is the class of the canonical line bundle, ([6], p. 223). It is well known that $\left(\tau \boldsymbol{R} P^{n}\right)=(n+1) \lambda$, where the parentheses indicate the stable isomorphism class of the tangent bundle determined by the standard smooth structure on $R P^{n},([6]$, p. 17). If $n$ is odd, let $g$ denote the composite

$$
\boldsymbol{R} P^{n} \stackrel{q}{\longrightarrow} C P^{[n / 2]} \stackrel{i}{\longrightarrow} B U(1),
$$

where $q$ is the canonical map, ([6], p. 223), and $i$ is the inclusion.

THEOREM 2.10. If $\boldsymbol{R} P^{n}$ is a branched covering of a $\pi$-manifold, then

$$
(n+1)(\lambda-1)=\sum_{i=1}^{m} g_{i}^{*} \eta_{k_{i}} .
$$

If the branch set is orientable, then there is a set $S \subset\{1,2,3, \cdots, m\}$ such that

$$
(n+1)(\lambda-1)=\sum_{i \in S} g^{*} \eta_{k_{i}}
$$

Proof. Formula (2.11) follows immediately from Theorem 2.6, Lemma 2.8, and the remarks above concerning $\left(\tau \boldsymbol{R} P^{n}\right)$ and $\widetilde{K O}\left(\boldsymbol{R} P^{n}\right)$. To establish (2.12), recall that $M S O(2)=B U(1)=K(Z, 2)$ and that homotopy classes of maps from $R P^{n}$ to $K(\boldsymbol{Z}, 2)$ are in one-to-one correspondence with $H^{2}\left(\boldsymbol{R} P^{n} ; \boldsymbol{Z}\right)=\boldsymbol{Z}_{2}$. Therefore, if one of the maps $g_{i}$ in (2.11) is essential, it is homotopic to $g$. The set $S$ is defined by the condition that $i \in S$ if and only if $g_{i}$ is essential. 
3. The proof of Theorem 1.1. The proof of Theorem 1.1 is organized into three short lemmas. As a preliminary, we review some elementary properties of the $K$-groups. There are two operations which connect the real and complex $K$-theories. The complexification operation, $\varepsilon_{u}: K O(X) \rightarrow K(X)$, which is induced by the operation of tensoring with $\boldsymbol{C}$ over $\boldsymbol{R}$, is a ring homomorphism and a $\lambda$-ring homomorphism and hence commutes with the Adams operations. The operation $\varepsilon_{0}: K(X) \rightarrow K O(X)$, which forgets complex structure, is a group homomorphism. If $\xi$ is any real bundle, then $\varepsilon_{0} \varepsilon_{u} \xi=2 \xi$, and if $\omega$ is any complex bundle, then $\varepsilon_{u} \varepsilon_{0} \omega=\omega \oplus \bar{\omega}$, ([6], p. 191).

LEMMA 3.1. If $g$ is the map (2.9), then

$$
g^{*} \eta_{k}=\left\{\begin{array}{l}
0, k \text { odd } \\
2(\lambda-1), k \text { even } .
\end{array}\right.
$$

Proof. Let $\zeta$ denote the canonical complex line bundle over $C P^{[n / 2]}$ or $B U(1)$. It is well known that $g^{*} \zeta=q^{*} \zeta=\varepsilon_{u} \lambda$, ([6], p. 223). It is easy to see that the class $\eta_{k}$ in $K O(M S O(2))$ corresponds to $\varepsilon_{0} \zeta-\mu_{k} \varepsilon_{0} \zeta$ in $\widetilde{K O}\left(C P^{[n / 2]}\right)$ via the homotopy equivalences $M S O(2)=$ $B S O(2)=B U(1)$ and the inclusion $i: C P^{[n / 2]} \rightarrow B U(1)$. One simply uses the definition of $\eta_{k}$, the facts that the homotopy equivalence $B U(1)=B S O(2)$ commutes with $\mu_{k}$ and that the Euler class of $\varepsilon_{0} \zeta$ is $c_{1}(\zeta)$, the generator of $H^{2}\left(C P^{[n / 2]} ; Z\right)$, ([6], p. 243). Therefore, we have

$$
g^{*} \eta_{k}=q^{*}\left(\varepsilon_{0} \zeta-\mu_{k} \varepsilon_{0} \zeta\right)=\varepsilon_{0} \varepsilon_{u} \lambda-\mu_{k} \varepsilon_{0} \varepsilon_{u} \lambda=2 \lambda-\mu_{k}(2 \lambda) .
$$

Now $2 \lambda=\lambda \oplus \lambda$ is an orientable bundle with classifying map induced by the map $Z_{2} \rightarrow O(2)$ which sends 1 into $\left(\begin{array}{rr}-1 & 0 \\ 0 & -1\end{array}\right)=$ rotation by $\pi$. So, (3.2) follows from (3.3) and the definition of $\mu_{k}$, ([2], p. 3).

In the proof of the next lemma, we will need to know the order, $c_{n}$, of the finite cyclic group $\widetilde{K O}\left(\boldsymbol{R} P^{n}\right)$. It is well known that $c_{n}=$ $2^{\varphi(n)}$, where $\varphi(n)$ is the number of integers in the set $\{s: 0<s \leqq n$, $s \equiv 0,1,2,4(\bmod 8)\}$, ([6], p. 223). An easy computation, ([6], p. 221), gives the following values for $c_{n}$, where $n$ is one of the values in the theorem of Brand,

$$
c_{n}=\left\{\begin{array}{l}
2^{2^{t-1}-1}, n=2^{t}-1, t \geqq 3, \\
2^{2^{t-1}+1}, n=2^{t}+1, t \geqq 2 .
\end{array}\right.
$$

Given a branched covering of $R P^{n}$ over a $\pi$-manifold, let $p$ be the number of distinct even exponents in a normalization of $f$ with 
essential classifying maps (2.5). In view of (2.12), (3.2) and (3.4), we have proven Lemma 3.5 below.

LEMMA 3.5. If $\boldsymbol{R} P^{n}$ is a branched covering of a $\pi$-manifold with orientable branch set, then

$$
p \equiv \frac{1}{2}(n+1)\left(\bmod \frac{1}{2} \text { order } \widetilde{K} O\left(R P^{n}\right)\right) .
$$

In particular,

$$
\begin{gathered}
p \equiv 2^{t-1}\left(\bmod 2^{2^{t-1}-2}\right), n=2^{t}-1, t \geqq 3, \\
p \equiv\left(2^{t-1}+1\right)\left(\bmod 2^{2^{t-1}}\right), n=2^{t}+1, t \geqq 2 .
\end{gathered}
$$

LEMma 3.9. If $\boldsymbol{R} P^{n}$ is a k-fold branched covering of a $\pi$-manifold with orientable branch set, and $n \neq 1,3$, or 7 , then

$$
\frac{1}{2}(n+1) \leqq p \leqq\left[\frac{1}{2} k\right] \text {. }
$$

Proof. The right-hand inequality is clear since any exponent in a normalization must be less than or equal to $k$ and the number of even numbers less than or equal to $k$ is smaller than $[(1 / 2) k]$. The left hand inequality is also clear because (3.7) and (3.8) clearly imply that $n=2^{t} \pm 1, n \neq 1,3$ or 7 , then $(1 / 2)(n+1)$ is the smallest possible representative of the congruence class of $p$ modulo $1 / 2$ order $\widetilde{K O}\left(\boldsymbol{R} P^{n}\right)$.

We are now ready to prove Theorem 1.1 by proving its contrapositive. The inequality (3.10) clearly implies that if $\boldsymbol{R} P^{n}$ is a branched covering of a $\pi$-manifold with orientable branch set and $n \neq 1,3$, or 7 , then the degree of the covering is greater than or equal to $n+1$. The left-hand side of (3.10) together with (3.7) and (3.8) imply that if $n \neq 1,3$, or 7 , then $p \neq 0,1$ and so the covering is not simple. Note that if $p=1$, then $n=1$ or 3 .

4. Adams operations and the brand characteristic classes. We remarked above that $\eta_{k}$ corresponds to the class $\varepsilon_{0} \zeta-\mu_{k} \varepsilon_{0} \zeta$ in $\widetilde{K O}(B U(1))$. Since $\varepsilon_{u}$ is a $\lambda$-ring homomorphism and $\mu_{k}$ can be expressed in terms of $\lambda$-ring operations and Adams operations ([1], p. 193), $\varepsilon_{u}$ commutes with $\mu_{k}$ and so $\varepsilon_{u} \eta_{k}=\varepsilon_{u} \varepsilon_{0} \zeta-\mu_{k} \varepsilon_{u} \varepsilon_{0} \zeta=(\zeta+\bar{\zeta})-$ $\left(\zeta^{k}+\bar{\zeta}^{k}\right)$. This means that it is possible to compute the action of the Adams operations on $\varepsilon_{u} \eta_{k}$ and produce formulas like the formulas for the action of the Steenrod operations on the Stiefel-Whitney classes, ([6], p. 245). We include these computations for the sake of completeness and in the hope that they will be useful in other 
contexts, since we will see below that these formulas provide no information on the characteristic classes in $\widetilde{K O}\left(\boldsymbol{R P} P^{n}\right)$.

In order to state our last proposition, we introduce some notation. If $x=\zeta+\bar{\zeta}$ and $y=\zeta^{k}+\bar{\zeta}^{k}$ and $j$ is an integer, the polynomial $\left(x^{j}-y^{j}\right) /(x-y)$ is symmetric in $\zeta$ and $\bar{\zeta}$ and consequently is the image of a polynomial in $\zeta$ under the operator $\varepsilon_{u} \varepsilon_{0}$. We denote this polynomial by $\theta(k, j)$. The proposition below follows easily from a little algebra and the fact that the Adams operation, $\psi^{j}$, has the property that $\psi^{j \zeta}=\zeta^{j}$.

Proposition 4.1. If $\psi^{j}$ is the Adams operation, then

$$
\begin{aligned}
& \psi^{j} \eta_{k} \equiv \eta_{k} \varepsilon_{0} \theta(k, j)-\sum_{i=1}^{\left[j^{\prime} 2\right]}\left(\begin{array}{c}
j \\
i
\end{array}\right) \psi^{j-2 i} \eta_{k}\left(\bmod k e r n e l \varepsilon_{u}\right), j \text { odd }, \\
& \psi^{j} \eta_{k} \equiv \eta_{k} \varepsilon_{0} \theta(k, j)-\sum_{i=1}^{j / 2-1}\left(\begin{array}{c}
j \\
i
\end{array}\right) \psi^{j-2 i} \eta_{k}\left(\bmod k e r n e l \varepsilon_{u}\right), j \text { even } .
\end{aligned}
$$

The action of the Adams operations on $\widetilde{K O}\left(\boldsymbol{R} P^{n}\right)$ is given by $\psi^{j}(\lambda-1)=\lambda-1, j$ odd, and $\psi^{j}(\lambda-1)=0, j$ even. The group $\widetilde{K}\left(\boldsymbol{R} P^{n}\right)$ is a finite cyclic group of order $2^{[n / 2]}$ and $\varepsilon_{u}: \tilde{K} \widetilde{O}\left(\boldsymbol{R} P^{n}\right) \rightarrow$ $\widetilde{K}\left(\boldsymbol{R} P^{n}\right)$ is always an epimorphism and is an isomorphism if $n \equiv 0,6$, $7(\bmod 8),\left([6]\right.$, p. 223). In particular, $\varepsilon_{u}$ is an isomorphism if $n=$ $2^{t}-1, t \geqq 3$, and $\varepsilon_{u}$ has kernel $Z_{2}$ if $n=2^{t}+1, t \geqq 2$. Therefore, not much is lost by complexifying and it is reasonable to suppose that the naturality $g^{*} \psi^{j} \varepsilon_{u} \eta_{k}=\psi^{j} g^{*} \varepsilon_{u} \eta_{k}$ might provide some information concerning the classes $g^{*} \eta_{k}$, since we can compute the righthand side using (3.2) and the left-hand side using (4.2) and (4.3). However, it is easy to see that $g^{*} \zeta^{r}=\varepsilon_{u} \lambda, r$ odd, and $g^{*} \zeta^{r}=\varepsilon_{u}(1)$, $r$ even, ([6], p. 234). This observation plus induction, counting the odd and even exponents in $\theta(k, j)$, and using the relation $\lambda^{2}=1$ in the ring $K O\left(R P^{n}\right)$, show that the naturality equation yields no new information.

5. Conclusion. It is clear that formula (3.10) says more about $R P^{n}$ as a branched covering of $S^{n}$ with oriental branch set than what is said in Theorem 1.1. For example, the fact that $p \neq 0$ if $n \neq 1,3$, or 7 says that high dimensional projective space cannot be a normalized branch cover of the sphere with orientable branch set and every exponent odd. The fact that $p \neq 0,1$, if $n \neq 1,3$, or 7 , rules out normalized covers where each partition associated with a component of $K$ has precisely one exponent greater than or equal to 2 and this exponent is the same for all partitions. Brand and Brumfiel [3] study $k$-fold branched coverings of this type where the 
partition of $k$ common to all components is the trivial partition of $k$. Such coverings generalize simple coverings where the singular set is connected and is all of $\widetilde{K}$. These observations and Theorem 1.1 constitute evidence for the conjecture that if $R P^{n}$ is a branched covering of $S^{n}$ with orientable branch set, then $n=1,3$, or 7 .

\section{REFERENCES}

1. J. F. Adams, On the groups $J(X) I$, Topology, 2 (1963), 181-195.

2. N. Brand, Necessary conditions for the existence of branched coverings, Invent. Math., 54 (1979), 1-10.

3. N. Brand and G. Brumfiel, Concordance classes of simple branched covers over $S^{2}$, $S^{3}$, and $S^{4}, I$, (preprint).

4. M. M. Cohen, A Course in Simple Homotopy Theory, New York: Springer-Verlag, 1970.

5. H. M. Hilden, Three-fold branched coverings of $S^{3}$, Amer. J. Math., 98 (1976), 989-997.

6. D. Husemoller, Fibre Bundles, New York: McGraw-Hill, 1966.

7. J. M. Montesinos, Three-manifolds as 3-fold branched covers of $S^{3}$, Quart. J. Math. Oxford Ser. (2) 27 (1976), 85-94.

8. P. Olum, Mappings of manifolds and the notion of degree, Ann. of Math., 58 (1953), 458-480.

9. C. T. C. Wall, Surgery on Compact Manifolds, London: Academic Press, 1970.

Received September 23, 1980.

UNIVERSITY OF HAWAII AT MANOA

HONOLULU, HI 96822 


\section{PACIFIC JOURNAL OF MATHEMATICS}

\section{EDITORS}

DONALD BABBITT (Managing Editor)

University of California

Los Angeles, California 90024

\section{Hugo Rossi}

University of Utah

Salt Lake City, UT 84112

C. C. MOore and Arthur AGuS

University of California

Berkeley, CA 94720
J. DugundJI

Department of Mathematics University of Southern California Los Angeles, California 90007

R. FinN and J. Milgram Stanford University Stanford, California 94305

ASSOCIATE EDITORS
R. ARENS
E. F. BECKENBACH
B. H. NeumanN
F. WOLF
K. YOSHIDA

\section{SUPPORTING INSTITUTIONS}

UNIVERSITY OF ARIZONA

UNIVERSITY OF BRITISH COLUMBIA

CALIFORNIA INSTITUTE OF TECHNOLOGY

UNIVERSITY OF CALIFORNIA

MONTANA STATE UNIVERSITY

UNIVERSITY OF NEVADA, RENO

NEW MEXICO STATE UNIVERSITY

OREGON STATE UNIVERSITY
UNIVERSITY OF OREGON

UNIVERSITY OF SOUTHERN CALIFORNIA

STANFORD UNIVERSITY

UNIVERSITY OF HAWAII

UNIVERSITY OF TOKYO

UNIVERSITY OF UTAH

WASHINGTON STATE UNIVERSITY

UNIVERSITY OF WASHINGTON 


\section{Pacific Journal of Mathematics}

Vol. 99, No. $1 \quad$ May, 1982

Mariano Giaquinta, Jindrich Necas, O. John and J. Stará, On the

regularity up to the boundary for second order nonlinear elliptic systems . . 1

Siegfried Graf, Realizing automorphisms of quotients of product $\sigma$-fields . . 19

Alfred Washington Hales and Ernst Gabor Straus, Projective colorings . . . 31

Sandra Hayes, The weak Nullstellensatz for finite-dimensional complex

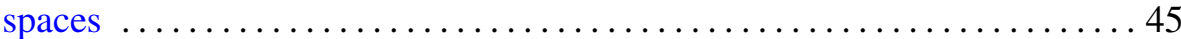

Gerald Norman Hile and Murray Harold Protter, The Cauchy problem

and asymptotic decay for solutions of differential inequalities in Hilbert

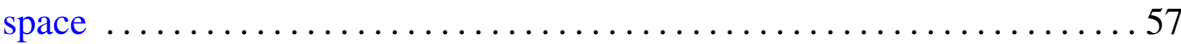

Robert D. Little, Projective space as a branched covering with orientable

branch set ......................................... 89

Jaroslav Mach, On the proximinality of Stone-Weierstrass subspaces . . . . . 997

John C. Morgan, II, On product bases ...................... 105

K. Balakrishna Reddy and P. V. Subrahmanyam, Altman's contractors

and fixed points of multivalued mappings . .................. 127

James Ted Rogers Jr., Decompositions of homogeneous continua . . . . . . . 137

Ahmed Ramzy Sourour, Characterization and order properties of

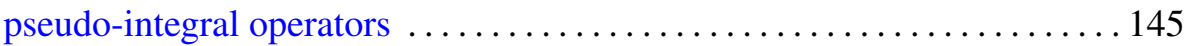

Robert Moffatt Stephenson Jr., Pseudocompact and Stone-Weierstrass

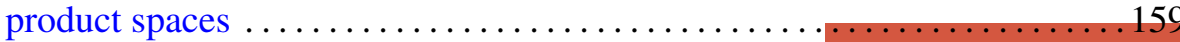

Bruce Stewart Trace, On attaching 3-handles to a 1-connected

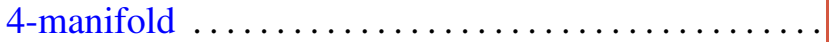

Akihito Uchiyama, The construction of certain BMO functions and the corona problem

Thomas Alva Whitehurst, An application of orthogonal polynomials to random walks ..............................

David J. Winter, Root locologies and idempotents of Lie and nonassociative algebras

William Robin Zame, The classification of uniform algebras on plane domains 